\title{
BASIC ALGEBRO-GEOMETRIC CONCEPTS IN THE STUDY OF PLANAR POLYNOMIAL VECTOR FIELDS
}

\author{
DANA SCHLOMIUK
}

\begin{abstract}
In this work we show that basic algebro-geometric concepts such as the concept of intersection multiplicity of projective curves at a point in the complex projective plane, are needed in the study of planar polynomial vector fields and in particular in summing up the information supplied by bifurcation diagrams of global families of polynomial systems. Algebro-geometric concepts are helpful in organizing and unifying in more intrinsic ways this information.
\end{abstract}

\section{Introduction and general discussion}

In the first announcement of the November 1996 meeting on the qualitative theory of planar vector fields, in Lleida, Spain, two goals for this meeting were mentioned: "On one hand to summarize the progress made in this field and on the other to explore new directions."

This article has two goals: first, to address some issues in response to the statement in the announcement to the Lleida meeting, in particular to appeal for more communication among specialists in this area and specialists in related areas; secondly, to show how very basic algebrogeometric concepts help in formulating results and in organizing the classification of low degree polynomial systems. A unified characterization of the bifurcation diagram of the quadratic Hamiltonian systems with a center in terms of the global geometry of systems serves as an illustration. The multiple singular cubic invariant curves as defined in section 4, govern the bifurcation diagram of this family of systems. A global affine invariant for this class is then used to compute the multiplicity of such curves. The article is addressed first to specialists on

This research was supported by NSERC and by the Quebec Education Ministry. 
planar polynomial vector fields. Work by such specialists as well as by specialists in the closely related areas, for example in the theory of foliations, acting as interface between the two areas (a beautiful example in this direction is given by C. Camacho in some of his work) as well as works acting as interface between planar polynomial vector fields and algebraic or analytic geometry, are of help in advancing the subject.

Planar polynomial vector fields appear often in applications and problems in this area can be stated in simple terms. However, progress has been slow and the area could perhaps get the price for the highest number of errors in the published literature, some of these made by excellent mathematicians. In view of this slippery ground (recently, in yet another published work a "theorem" turned out to have a flawed proof), we clearly need to pay great attention to rigour and repeat calculations using alternative ways whenever possible. Also, viewing problems from different angles and establishing connections with other areas of mathematics can only be helpful.

Perhaps the best result in this area which was obtained in the past ten years is the proof of the finiteness theorem. This theorem appeared first in a paper published in 1923 by Dulac [10], who claimed to have proved that any planar polynomial vector field has a finite number of limit cycles. It took almost 60 years to realize that this proof is not correct and more years to find a proof of this theorem. We now have two proofs, one of Il'yashenko [17] and another one of Ecalle [13], and the results are also applicable to analytic vector fields on the sphere. There is at least one other theorem which was first put on paper as a theorem but for which it took a long time to have a proof: this is Fermat's last theorem, which was stated by Fermat around 1637. After 357 years since Fermat wrote in his margin of his copy of Gauss' Disquisitiones Arithmeticae that he has a proof which that margin was too small to contain, in 1994, Andrew Wiles and Richard Taylor proved a theorem on semi-stable elliptic curves which as Ribet showed, implies Fermat's last theorem.

Fermat's problem and Hilbert's 16th problem have something in common: they both appeal by their simplicity and clarity of statement. Due to this, many people became aware of Fermat's last theorem and when Wiles proved it, the news made it to the front page of the New York Times. As mathematicians we are even more interested in this proof. Lots of articles appeared about in and lots of lectures were given. A beautiful lecture was given in 1993 in Canada by Barry Mazur at a joint meeting of the A.M.S. and C.M.S. and a videotape of this lecture [22] was made.

We understand that the progress on Fermat's theorem was achieved 
through a truly broad view and many deep mathematical connections. The role of connections was expressed very well in an address of Armand Borel [3]:

"In fact, I would rather schematize the structure of mathematics by a complicated graph, where the vertices are the various parts of mathematics and the edges describe the connections between them. These connections sometimes go one way, sometimes both ways, and the vertices can act both as sources and sinks. The development of the individual topics is of course the life and blood of mathematics, but, in the same way as a graph is more than the union of its vertices, mathematics is much more than the sum of its parts. It is the presence of those numerous, sometimes unexpected edges, which makes mathematics a coherent body of knowledge, and testifies to its fundamental unity, in spite of its being too vast to comprehend by one single mind."

We may view planar or more generally surface vector fields as a vertex and edges to and from this vertex play an important role.

In 1922, about the time when Dulac was putting his finishing touches on his paper "Sur les cycles limites" which appeared in 1923, Mordell stated the conjecture which bears his name (like Dulac's conjecture this is a finiteness conjecture): "any given curve of genus $g \geq 2$ has only a finite number of rational points. Mordell's conjecture became Faltings' theorem in 1983 and later Faltings received the Fields medal for his work, a major achievement in diophantine geometry which had implications for the proof of Fermat's last theorem.

Elliptic curves played a crucial role in the proof of Fermat's last theorem. Andrew Wiles and Richard Taylor's result is about elliptic curves: "Every semistable elliptic curve is modular" but this result, as K. Ribet showed, implies Fermat's last theorem. This is made possible by connections among the theory of elliptic curves, group representations, and the theory of modular forms.

The story of Fermat's last theorem is fascinating by the beautiful mathematical ideas and the deep connections which link them together. It pays off to have a wide viewpoint. In particular, if we now turn to our problems on planar vector fields and more specifically to quadratic vector fields and Hilbert's 16th problem for this case, it could only be of help if we were to have a broader viewpoint and to accompany our specific calculations with explicit connections to related areas of mathematics. This is done in the best works in the subject but is it the rule? The two proofs of Il'yashenko [17] and Ecalle $[\mathbf{1 3}]$ of the finiteness theorem are both securely linked with other areas of mathematics. Il'yashenko's proof is the one better understood because it is based in more familiar 
ground: on complex analysis and in particular the Phragmen-Lindelöf principle. Ecalle's proof has a very different flavour. Ecalle makes the point that his proof is constructive. He insists on the broader scope of the methods employed (the theory of resummation; he considers this proof an exercise in "resummation") and on the fact that they lead to a constructive approach. Ecalle's proof is not yet completely understood by the specialists. Nevertheless, its more constructive character and virtual links with non-commutative geometry, make this proof very attractive and interesting.

Let us now focus our attention on planar polynomial systems and in particular on quadratic or cubic ones. There are numerous articles on planar quadratic or cubic vector fields. Some of these deal with perturbations of singular cycles of such systems. In two interesting articles [11], [12], the authors describe a method for solving the finiteness part (which the authors call "the existential part") of Hilbert's 16th problem for quadratic systems and which we state as follows: Prove that $H(n)$ is finite where by $H(n)$ it is meant the lowest cardinal number which bounds the number of limit cycles for any polynomial vector field of degree $n$. Hilbert's 16th problem is to find $H(n)$ as well as to establish the relative positions of limit cycles. In the first one of these articles it is shown that to solve the finiteness part of Hilbert's 16th problem one needs to prove the finite cyclicity of 121 cases of singular cycles. While a number of these cases are done, many still remain open and the program could well take years to accomplish. Considering that in the past 97 years no examples could be found for which more than four limit cycles could be proven to exist, not only is it conjectured that $H(2)<\infty$ but actually that $H(2)=4$. If one is interested in structure, even complete proofs of finite cyclicity for all the 121 cases of graphic cycles are not enough help but the program outlined in [11], [12] is interesting because of the specific study of the return maps involved. However, attempting to get a global understanding for larger and larger classes of quadratic systems in an organized way would lead to more information about these systems and eventually to exact bounds for these classes. Since the bifurcation diagram of the quadratic systems must have a very large number of distinct phase portraits, conceptual unifying themes or guiding principles through this labyrinth are essential. Looking for intrinsic ways of characterizing the bifurcation locus whenever possible without constantly refering to coordinates is a legitimate goal.

The parameter space where these bifurcation diagrams sit is $\mathbb{R}^{12}$ but on the space acts the group of affine coordinate transformations and positive time rescaling. Thus, the parameter space is actually five dimensional. Some of the hypersurfaces of the bifurcation locus are algebraic, others 
are analytic and still others are not even be analytic.

In a short, very nice article [8] on quadratic systems written in 1966, W. A. Coppel wrote:

"Ideally one might hope to characterize the phase portraits of quadratic systems by means of algebraic inequalities on the coefficients. However, attempts in this direction have met with limited success..."

Now, more than 30 years later, it is known that algebraic inequalities would not suffice. Analytic as well as non-analytic ones are necessary. Even if all these inequalities are included, more intrinsic means for classifying systems would need to be developped for the classification to be more illuminating. In classifying mathematical objects, simpler mathematical objects or concepts are used: topological spaces are classified by their homotopy or homology groups, algebraic curves are birationally classified by their genus, $C^{*}$-algebras by their $K$-theory groups, etc.

Dynamical systems are a mixed breed, having both geometric and analytic features. In view of the thousands phase portraits and of their associated inequalities it is perhaps not completely idle to try to find better ways of classifying systems or of encoding information. We do have ways of attaching invariants to systems. The whole theory of normal forms attempts to attach invariants to systems. To piece together the local and nonlocal informations into global ones is a lot more difficult because the analysis of the Poincaré return map for families of systems is so hard to perform.

Works on classifying systems, in particular on quadratic or cubic ones, may benefit by use of some knowledge in algebraic geometry. The very powerful methods used in this field benefitted the theory of partial differential equations as one could see for example on the works on the $K d V$ equation. Algebraic geometry has many concepts which can be used in this area and furthermore it has method which may be of help in inspiring better organization in classification works than what is available in the literature.

In this work we show how very basic algebro-geometrical concepts can be used for problems on planar polynomial vector fields. The paper is organized as follows: section 2 includes some problems and results on polynomial vector fields which have a clear algebro-geometric character. This section is by no means intended as a quick survey but only as an illustration of such problems. The literature contains works of this nature and the section refers to some of them. In section 3 we discuss a very basic concept of algebraic geometry, the concept of intersection multiplicity of projective curves and introduce several multiplicities for planar polynomial vector fields expressed in terms of this concept. In section 4 
we show how the concepts introduced in the previous section can be used for encoding in an intrinsic and compact way, informations about some families of planar polynomial systems. As an illustration we consider the family of quadratic Hamiltonian systems with a center, example which is chosen because of its simplicity. This family is algebraically integrable with cubic polynomial first integrals but an analogous discussion can be carried out when we treat other families of planar quadratic systems when classifying their singularities, regardless if these families are integrable or not. Such a discussion must be based on numerous calculations and other examples such as for the class of quadratic vector fields with a weak focus (work for this class is in progress [25]), or of other classes of nonintegrable systems will be given elsewhere. We also show on an example how statements of results can gain in clarity and in geometric meaning by using the concepts introduced in section 3. Some questions for future work are raised.

\section{Problems on polynomial vector fields which have a clear algebro-geometric content}

Let $v(x, y)=P(x, y) \partial / \partial x+Q(x, y) \partial / \partial y$ be a real polynomial vector field and consider the associated differential systems:

$$
\begin{aligned}
& d x / d t=P(x, y) \\
& d y / d t=Q(x, y)
\end{aligned}
$$

A curve $f(x, y)=0$ with $f$ a polynomial with complex coefficients is an invariant algebraic curve for $v(x, y)$ or for a system given by equation (2.1), if for some polynomial $K$ with complex coefficients we have $v f=f K$. If $f$ is an irreducible polynomial over $\mathbb{C}$ we say that the curve is an algebraic solution of the system. We say that the real (respectively complex) system given by equation (2.1) is algebraically integrable if and only if there exists a rational function $R(x, y)=p_{1}(x, y) / p_{2}(x, y), p_{i}$ a polynomial with real (respectively complex) coefficients, $i=1,2$, which is a first integral of the system. We say that the systems has a Darboux [9] first integral (resp. Darboux integrating factor) if the system has a first integral (resp. integrating factor) of the form $f_{1}^{\lambda_{1}} f_{2}^{\lambda_{2}}, \ldots, f_{n}^{\lambda_{n}}$ where $f_{i}$ are algebraic solutions of the system and $\lambda_{i}$ are complex numbers [29].

Theorem 2.1 (Darboux [9] and Jouanolou [18]). For a system given by equation (2.1) with $P, Q \in \mathbb{C}[x, y]$ such that $m=$ $\max (\operatorname{deg}(P), \operatorname{deg}(Q))$ and such that $f_{i}=0, i=1, \ldots, q$ are distinct algebraic solutions for the system, with $q \geq m(m+1) / 2$ we either have a 
Darboux first integral or a Darboux integrating factor. Furthermore we have:

1) If $q \geq m(m+1) / 2+1$, the system has a Darboux first integral.

2) If $q \geq m(m+1) 2+2$, the system has a first integral which is a rational function over $\mathbb{C}$.

Poincaré's problem on algebraic integrability [27], [28] is to recognize when a polynomial system given by equation (2.1) is algebraically integrable.

Related to the above problem is the following one:

Give a bound for the degree of an invariant algebraic curve which a system given by equation (2.1) could have.

Painlevé's problem [23], [24] is the problem of recognizing if a system given by equation (2.1) has a general integral

$$
p_{1}(x, y)-K p_{2}(x, y)=0
$$

where $K$ is a constant and $p_{i}$ are polynomials over $\mathbb{C}$, which is an algebraic curve of a given genus.

Clearly all of these problems as well as the problem of determining when systems have invariant algebraic curves have an algebro-geometric content and the literature contains works dealing with problems on foliations or on systems given by equation (2.1) which have a clear algebrogeometric content $[\mathbf{4}],[\mathbf{6}],[\mathbf{7}],[\mathbf{9}],[\mathbf{1 5}]$ and $[\mathbf{1 8}]$.

Other problems like the one of distinguishing between a center and a focus turn out to have some algebro-geometric components. In the quadratic case the conditions for the center turned out to have a complete algebro-geometric content [31], but the situation is more complex in the case of higher degree vector fields.

\section{Multiplicity of intersection of projective curves and other concepts as basic tools in studying planar polynomial vector fields}

The singularities of polynomial vector fields $P \partial / \partial x+Q \partial / \partial y$ are the common points of the curves $P(x, y)=0$ and $Q(x, y)=0$. Counting these points with their respective multiplicities adds information. For these multiplicities, the full projective completions $P^{*}(X, Y, Z)=0$, $Q^{*}(X, Y, Z)=0$ (here $P^{*}(X, Y, Z)=Z^{n} P(X / Z, Y / Z), Q^{*}(X, Y, Z)=$ $Z^{n} Q(X / Z, Y / Z)$, if $\left.n=\operatorname{deg}(P)=\operatorname{deg}(Q)\right)$ of the curves $P=0$ and $Q=0$ are needed. For example, whenever the projective completions 
$P^{*}=0$ and $Q^{*}=0$ of these curves have a point in common at infinity, the systems will have fewer singular points in the finite plane. If the curves have a higher contact at infinity, this will be reflected in the number of points in the finite plane which will be fewer. It is important to count these points over $\mathbb{C}$. Roughly speaking the intersection multiplicity of two algebraic curves at a point indicates how many points the curves have in common at that point. For example the line $y=0$ and the parabola $y-x^{2}=0$ intersect at the origin with intersection multiplicity two, and the line $y=0$ and the cubic $y-x^{3}=0$ intersect at the origin with intersection multiplicity three, the three intersection points coinciding in this case at $(0,0)$.

We have several ways of defining the concept of multiplicity of intersection of algebraic curves: via resultants [19] of homogeneous polynomials in three variables over $\mathbb{C}$, via the local rings [14], axiomatically, etc.

Using resultants of polynomials in three variables [19], we can define multiplicity of intersection at a point $p=[a: b: c]$ of two projective curves given by the equations $F(X, Y, Z)=0$ and $G(X, Y, Z)=0$, where $F$ and $G$ are homogeneous polynomials over $\mathbb{C}$, without common nontrivial factors as follows: Assume these curves to be placed in general position with respect to $Z$ for example, i.e. the degree of $F$ (resp. $G$ ) equals the degree of the highest order term containing only $Z$ in $F$ (resp. $G$ ), then we can eliminate $Z$ by considering the resultant $R(X, Y)[\mathbf{1 9}]$ of the polynomials $F, G$, with respect to $Z$. Since $F$ and $G$ have no nontrivial common factors, $R(X, Y)$ is a nontrivial homogeneous polynomial [14] in $X, Y$ of degree $n . m$ where $n=\operatorname{deg}(F), m=\operatorname{deg}(G)$ and hence $R(X, Y)$ splits into n.m linear factors over $\mathbb{C}$, perhaps not all of them distinct. If $p=[a: b: c]$ is a point of intersection of $F=0$ with $G=0$, then $(a, b)$ is a solution of $R(X, Y)=0$ and $c$ is a common solution of the equations $F(a, b, Z)=0$ and $G(a, b, Z)=0$. We say that the point $[a: b: c]$ is a multiple point of intersection of $F=0$ with $G=0$ of multiplicity $k$ if $(a, b)$ is a solution of one of the linear factors in $R(X, Y)$ appearing there with the multiplicity $k$.

A definition which generalizes easily for intersection multiplicity of several curves at a point is via the local rings $[\mathbf{1 4}]$ :

Definition 3.1. The intersection multiplicity $I_{p}\left(F_{1}, F_{2}\right)$ at a point $p$ of the algebraic curves $C_{1}, C_{2}$ in $\mathbb{C}^{2}$ where $C_{i}: F_{i}(x, y)=0$ is defined as being zero if the curves do not intersect, infinity if the curves have a common component and otherwise we use the definition

$$
I_{p}\left(F_{1}, F_{2}\right)=\operatorname{dim}_{\mathbb{C}} O_{p} /\left(F_{1}, F_{2}\right)
$$

where $O_{p}$ is the local ring of the affine complex plane $A^{2}(\mathbb{C})=\mathbb{C}^{2}$ at $p$, 
i.e. $O_{p}$ is the ring of germs of rational functions $q(x, y) / r(x, y)$ which are defined at $p$, i.e. such that $r(p) \neq 0$.

We are interested in planar polynomial vector fields

$$
v(x, y)=P(x, y) \partial / \partial x+Q(x, y) \partial / \partial y
$$

and their associated differential systems

$$
\begin{aligned}
& d x / d t=P(x, y), \\
& d y / d t=Q(x, y)
\end{aligned}
$$

where $P(x, y), Q(x, y)$ are polynomials with real coefficients.

When studying such systems, the compactification of the systems on the sphere is usually used. We may consider the plane $x, y$ as being $Z=1$ in the space $\mathbb{R}^{3}$ and project the plane via the central projection onto the sphere. This compactifies the plane to the upper hemisphere completed by the equator. The central projection of the vector field yields an analytic vector field on the sphere [16].

To the vector field given by equation (3.2) there is an associated direction field on $\mathbb{R}^{2}$ and a differential equation $P d y-Q d x=0$.

Let us suppose that $P$ and $Q$ have no nonconstant common factor. We consider the associated differential 1-form $\omega_{1}=Q(x, y) d x-P(x, y) d y$ and the differential equation $\omega_{1}=0$. The affine plane $\mathbb{R}^{2}$ (respectively $\mathbb{C}^{2}$ ) is compactified to the real (resp. complex) projective space $\mathbb{R} P(2)=$ $\left(\mathbb{R}^{3}-\{0\}\right) / \sim,\left(\right.$ resp. $\left.\mathbb{C} P(2)=\left(\mathbb{C}^{3}-\{0\}\right) / \sim\right)$, where $(x, y, z) \sim\left(x^{\prime}, y^{\prime}, z^{\prime}\right)$ if and only if $\left(x^{\prime}, y^{\prime}, z^{\prime}\right)=u(x, y, z)$ for some $u \neq 0, u$ real (resp. complex). Let $[X: Y: Z]$ be the equivalence class of $(X, Y, Z)$. Clearly the restriction of the equation $\omega_{1}=0$ on nonsingular points of the equation $P d y-Q d x=0$, defines a foliation on this submanifold of $\mathbb{C}^{2}$. Thus a foliation with singularities on $\mathbb{C}^{2}$ is obtained. This foliation can be extended to a singular foliation on $\mathbb{C} P(2)$ and the one-form $\omega_{1}$ can be extended to a meromorphic one-form on $\mathbb{C} P(2)$ [5]. Analogously to the way we can describe a plane projective curve by a single homogeneous equation in $x, y, z$ we can describe this meromorphic one-form by a single one-form $A^{*}(X, Y, Z) d X+B^{*}(X, Y, Z) d Y+C^{*}(X, Y, Z) d Z$ with homogeneous polynomial coefficients. Indeed, let us suppose that $P$ and $Q$ have no common nontrivial factors and $\max (\operatorname{deg}(P), \operatorname{deg}(Q))=n>0$ and consider the application $r: \mathbb{C}^{3} \backslash\{(X, Y, Z) \mid z=0\} \rightarrow \mathbb{C}^{2}$ given by $r(X, Y, Z)=(X / Z, Y / Z)$. From the relations $x=X / Z, y=Y / Z$ it follows that $d x=(Z d X-X d Z) / Z^{2}, d y=(Z d Y-Y d Z) / Z^{2}$. The differential form $\widetilde{\omega}=r^{*}\left(\omega_{1}\right)$ has poles at $Z=0$ and the equation $\omega_{1}=0$ can be written in coordinates $X, Y, Z$ as follows:

$$
\begin{aligned}
\widetilde{\omega}=Q(X / Z, Y / Z)(Z d X- & X d Z) / Z^{2} \\
& -P(X / Z, Y / Z)(Z d Y-Y d Z) / Z^{2}=0 .
\end{aligned}
$$


Then $\omega=Z^{n+2} \widetilde{\omega}$ has polynomial coefficients of degree $n+1$ and for $Z \neq 0$, the equations $\omega=0$ and $\widetilde{\omega}=0$ have the same solutions. The differential equation $\omega=0$ is:

$Z^{n} Q(X / Z, Y / Z)(Z d X-X d Z)-Z^{n} P(X / Z, Y / Z)(Z d Y-Y d Z)=0$.

Hence, regrouping the terms in $d X, d Y, d Z$ respectively, we have:

$$
\begin{aligned}
\omega=Z Q^{*}(X, Y, Z) d X-Z P^{*} & (X, Y, Z) d Y \\
& +\left(Y P^{*}(X, Y, Z)-X Q^{*}(X, Y, Z)\right) d Z
\end{aligned}
$$

where

$$
\begin{aligned}
& P^{*}(X, Y, Z)=Z^{n} P(X / Z, Y / Z), \\
& Q^{*}(X, Y, Z)=Z^{n} Q(X / Z, Y / Z) .
\end{aligned}
$$

We have thus obtained a polynomial one-form

$$
\omega=A^{*}(X, Y, Z) d X+B^{*}(X, Y, Z) d Y+C^{*}(X, Y, Z) d Z
$$

with

$$
\begin{aligned}
& A^{*}=Z Q^{*}, \\
& B^{*}=-Z P^{*}, \\
& C^{*}=Y P^{*}-X Q^{*}
\end{aligned}
$$

hence $A^{*}, B^{*}, C^{*}$ are homogeneous polynomials of degree $n+1$ in the variables $X, Y, Z$. The form $\omega$ yields an equation $\omega=0$ on $\mathbb{C}^{3}$. Just as planar projective curves are described by using one chart in $\mathbb{C}^{3}$ and one homogeneous polynomial equation in three variables, we can use homogeneous coordinates $[X: Y: Z]$ and just one differential equation

$$
L(X, Y, Z) d X+M(X, Y, Z) d Y+N(X, Y, Z) d Z=0
$$

with homogeneous polynomials in $(X, Y, Z)$ of the same degree as coefficients, to obtain a differential equation on $\mathbb{C} P(2)$ provided the condition $L X+M Y+N Z=0$ is satisfied [9]. In particular for the equation $\omega=0$ to yield an equation on $\mathbb{C} P(2)$ we need to verify the condition $A^{*} X+B^{*} Y+C^{*} Z=0$. This condition clearly holds.

The singularities of the foliation induced by $\omega_{1}$ on $\mathbb{C} P(2)$ are the intersection points of the curves $A^{*}=0, B^{*}=0, C^{*}=0$ and these points need to be counted with multiplicities of intersection. The concept of intersection multiplicity above extends to that of intersection multiplicity of several curves at a point of the projective plane. 
Definition 3.2. The intersection multiplicity $I_{p}\left(f_{1}, f_{2}, \ldots, f_{n}\right)$ at a point $p$ of the algebraic curves $C_{1}, C_{2}, \ldots, C_{n}$ in $\mathbb{C}^{2}$ where $C_{i}: f_{i}(x, y)=0$ is defined as being zero if the curves do not intersect, infinity if the curves have a common component and otherwise we use the definition

$$
I_{p}\left(f_{1}, f_{2}, \ldots, f_{n}\right)=\operatorname{dim}_{\mathbb{C}} O_{p} /\left(f_{1}, f_{2}, \ldots, f_{n}\right) .
$$

In particular we shall be interested in the way the curves $A^{*}=0, B^{*}=0$ and $C^{*}=0$ intersect and hence in the values of $I_{p}\left(A^{*}, B^{*}, C^{*}\right)$ for $p$ in the projective plane.

\section{Notation 3.1.}

$$
m(p)=I_{p}\left(A^{*}, B^{*}, C^{*}\right)
$$

This multiplicity is computed by using an appropiate chart and the Definition 3.2. The value of $m(p)$ is independent of the particular chart.

We have the canonical imbedding of the affine plane $A^{2}(\mathbb{R})=\mathbb{R}^{2}$ (resp. $\left.A^{2}(\mathbb{C})=\mathbb{C}^{2}\right)$ into the real (resp. complex) projective plane

$$
i: A^{2}(\mathbb{R}) \longrightarrow \mathbb{R} P(2), \quad(i(x, y)=[x: y: 1]) .
$$

We shall identify a point $p$ in the affine plane with its image $i(p)$ in the projective plane. Vector fields on $A^{2}(\mathbb{R})$ (resp. $A^{2}(\mathbb{C})$ ) yield Pfaff forms and foliations on $\mathbb{R} P(2)$ (resp. $\mathbb{C} P(2)$ ).

Proposition 3.1. If $p$ is a finite or infinite singular point of a polynomial system (3.2) and $A^{*}, B^{*}, C^{*}$ are as defined before in equation (3.6), we have:

$$
\begin{gathered}
I_{p}\left(A^{*}, B^{*}, C^{*}\right)=I_{p}\left(Z, Y P^{*}-X Q^{*}\right)+I_{p}\left(P^{*}, Q^{*}\right) . \\
m(p)= \begin{cases}I_{p}\left(P^{*}, Q^{*}\right)\left(=I_{p}(P, Q)\right) & \text { if } p \text { is finite } \\
I_{p}\left(Z, Y P^{*}-X Q^{*}\right)+I_{p}\left(P^{*}, Q^{*}\right) & \text { if } p \text { is infinite. }\end{cases}
\end{gathered}
$$

The proof follows easily from the fact that $Z$ is a common factor of $A^{*}$ and $B^{*}$ and from basic properties of intersection multiplicities [19].

Corollary 3.1. $I_{p}\left(A^{*}, B^{*}, C^{*}\right) \geq I_{p}\left(P^{*}, Q^{*}\right), I_{p}\left(Z, Y P^{*}-X Q^{*}\right)$.

$$
I_{p}\left(A^{*}, B^{*}, C^{*}\right)>I_{p}\left(P^{*}, Q^{*}\right) \longleftrightarrow p \in\left\{[X: Y: Z] \mid Y P^{*}-X Q^{*}=0=Z\right\}
$$


and if $p$ is a point at infinity, then we have:

$$
I_{p}\left(A^{*}, B^{*}, C^{*}\right)>I_{p}\left(Z, Y P^{*}-X Q^{*}\right) \longleftrightarrow I_{p}\left(P^{*}, Q^{*}\right) \geq 1 .
$$

\section{Notation 3.2.}

$$
m_{\infty}(p)=I_{p}\left(Z, Y P^{*}-X Q^{*}\right) .
$$

$m(p)$ indicates the number of singular points finite or infinite which can bifurcate from $p$ in a quadratic perturbation, $I_{p}\left(P^{*}, Q^{*}\right)$ indicates the number of finite singular points which can bifurcate from $p$ under a quadratic perturbation and $m_{\infty}(p)$ indicates the total number of infinite singular points, which can bifurcate from $p$.

Definition 3.3. We call global finite multiplicity $m_{f}$ the number

$$
m_{f}=\sum_{p \in A^{2}}\left(I_{p}(P, Q)\right) .
$$

The multiplicities $I_{p}(P, Q), I_{p}\left(P^{*}, Q^{*}\right)$ and $m_{\infty}(p), m(p), m_{f}$ are basic tools in classifying planar polynomial systems (3.2).

Clearly we have:

For a nonlinear polynomial system given by equation (3.2) with $\max (\operatorname{deg}(P), \operatorname{deg}(Q))=n$ and with $P, Q$, without common nontrivial factors, in view of Bézout's theorem [14], [19], we clearly have:

$$
I_{p}\left(P^{*}, Q^{*}\right), \quad I_{p}(P, Q) \leq n^{2}, \quad m_{\infty}(p) \leq n+1,
$$

and hence $m(p) \leq n^{2}+n+1$. We also have:

Proposition 3.2 (Darboux [9]). For a polynomial system given by equation (3.2) with $\operatorname{deg}(P)=\operatorname{deg}(Q)=n>0$ and with $P, Q$ without nontrivial common factors and $A^{*}, B^{*}, C^{*}$ as in equation (3.6) and (3.5), we have:

$$
\sum_{p \in \mathbb{C} P(2)} I_{p}\left(A^{*}, B^{*}, C^{*}\right)=n^{2}+n+1 .
$$

Just as the points at infinity of affine curves gain equal status with points in the finite plane by their inclusion in the projective plane where all lines are treated equally, the singularities at infinity of the vector fields gain equality of status when viewed as points of the projective 
plane, forgetting when it is convenient for unifying purposes their special status as points on the invariant line at infinity.

The intersection multiplicity of projective curves comes in handy when studying families of planar quadratic vector fields. For example, the various multiplicities defined before are useful when classifying families of polynomial systems which are algebraically integrables, when dealing with systems which have invariant algebraic curves or when classifying singularities in any family of either integrable or not integrable systems. Some of their bifurcation surfaces are given in terms of these concepts.

\section{Applications}

We are interested in global properties of polynomial systems of a given degree $n$ and more specifically in quadratic systems. The group of affine transformations acts on such a class. Ideally we would like to have a complete set of global invariants for this class of systems. This is of course a far shot as this class has a very large numver of phase portraits. We start here with a very simple subclass: the class of quadratic Hamiltonian systems with a center. This is an algebraic class (all systems have a cubic polynomial as first integral) and we may regard it as "trivial". Still, this is the place to start our search. Indeed, if we look up the literature on classifying quadratic systems, we see a mass of phase portraits and sometimes, though not always, bifurcation diagrams. Apart from performing calculations of phase portraits and of writing up directories of inequalities, an effort needs to be made to "sum up" these calculations and for this it is absolutely necessary to merge knowledge from several areas of mathematics: bifurcation theory, foliation theory, some algebraic geometry, etc. It seems to us that an effort to piece together global informations for quadratic systems with the help of local invariants or of the work done by specialists on the theory of foliations is necessary. We mentioned in the introduction that polynomial vector fields constitute a slippery research subject. We do want to move on solid ground and to start this initial work with an important nonintegrable class, having a very large number of phase portraits before attempting the study of one of the simplest classes was not recommendable.

However, work is now in progress along these lines for the (generically nonintegrable) class of quadratic systems with a weak focus and results will appear elsewhere. Our analysis of the quadratic Hamiltonian systems with a center shows us that at all the points of the bifurcation locus the same type of global phenomenon occurs, regardless of the specific local or nonlocal type of bifurcation (of singularities at infinity, of singularities in the finite plane or of saddle to saddle connections): 
the presence in the systems of multiple (in a sense to be defined below) invariant algebraic curves or equivalently, the presence of curves with multiplicity indices with respect to the systems greater than one. This shows that it is possible to piece together in a unified way the various bifurcations phenomena into something which is global and stimulates us to look for analogous results in more general, non integrable situations. We shall first introduce an affine invariant for systems $S$ given by equations (3.3) which posses an invariant algebraic curve $C: f(x, y)=0$. We shall denote such a structured dynamical system by $(S, f)$.

Definition 4.1. Let us suppose that for a real system $S$ given by equations (3.3), $P$ and $Q$ have no nonconstant common factors and the system has an algebraic invariant curve $C: f(x, y)=0$. We consider the divisor $D(S, f)$ of the curve $C$, defined as follows:

$$
D(S, f)=\sum_{\substack{p \in \operatorname{Sing}(\omega) \\ p \in \widetilde{C}}} I_{p}\left(P^{*}, Q^{*}\right) p
$$

where $P^{*}, Q^{*}$ are the polynomials defining the projective completions of the curves $P=0, Q=0, \widetilde{C}$ is the projective completion of the curve $C$ and $\omega$ is defined as before in equation (3.5), (3.6).

We define a multiplicity of the invariant curve $C$ with respect to a system $S$ (or a vector field $v$ ) given by equation (3.3) and denote it by $\mu(C, S)$ the degree of this divisor i.e.:

$$
\mu(C, S)=\sum_{\substack{p \in \operatorname{Sing}(\omega) \\ p \in \widetilde{C}}} I_{p}\left(P^{*}, Q^{*}\right)
$$

Sometimes the shorthand $\mu(C)$ will be used for $\mu(C, S)$.

Proposition 4.1. $\mu(C, S)$ is an affine invariant of the structured $d y$ namical system $(S, f)$. 
Proof: Let us consider a real affine transformation $T\left(x^{\prime}, y^{\prime}\right)=(x, y)$ given by a two by two real invertible matrix $A=\left(\left(a_{i j}\right)\right)$ and column vector $B=\left(\left(b_{i}\right)\right), i=1,2$ :

$$
\left(\begin{array}{l}
x \\
y
\end{array}\right)=A\left(\begin{array}{l}
x^{\prime} \\
y^{\prime}
\end{array}\right)+B
$$

We also consider the corresponding projective transformation $T^{\prime}\left(X^{\prime}, Y^{\prime}, Z^{\prime}\right)=(X, Y, Z)$

$$
\left(\begin{array}{l}
X \\
Y \\
Z
\end{array}\right)=A\left(\begin{array}{l}
X^{\prime} \\
Y^{\prime} \\
Z^{\prime}
\end{array}\right)
$$

where

$$
A^{\prime}=\left(\begin{array}{ccc}
a_{11} & a_{12} & b_{1} \\
a_{21} & a_{22} & b_{2} \\
0 & 0 & 1
\end{array}\right)
$$

Then we have:

$$
\left(\begin{array}{l}
x^{\prime} \\
y^{\prime}
\end{array}\right)=A^{-1}\left(\begin{array}{l}
P^{T} \\
Q^{T}
\end{array}\right)=(1 / d)\left(\begin{array}{r}
a_{22} P^{T}-a_{12} Q^{T} \\
-a_{21} P^{T}+a_{11} Q^{T}
\end{array}\right)
$$

where $P^{T}\left(x^{\prime}, y^{\prime}\right)=P\left(a_{11} x^{\prime}+a_{12} y^{\prime}, a_{21} x^{\prime}+a_{22} y^{\prime}\right), Q^{T}\left(x^{\prime}, y^{\prime}\right)=P\left(a_{11} x^{\prime}+\right.$ $\left.a_{12} y^{\prime}, a_{21} x^{\prime}+a_{22} y^{\prime}\right)$ and $d=\operatorname{det}(A)$. Let $P^{\prime}=(1 / d)\left(a_{22} P^{T}-a_{12} Q^{T}\right)$, $Q^{\prime}=(1 / d)\left(-a_{11} P^{T}+a_{21} Q^{T}\right)$. We denote by $P^{\prime *}, Q^{\prime *}$ the homogeneous polynomials in $x^{\prime}, y^{\prime}, z^{\prime}$ obtained from $P^{\prime}, Q^{\prime}$. Let $p^{\prime}$ be such that $T^{\prime}\left(p^{\prime}\right)=p$. We have:

$$
I_{p^{\prime}}\left(P^{\prime *}, Q^{\prime *}\right)=I_{p^{\prime}}\left(\left(a_{22} P^{*}-a_{12} Q_{22}^{*}\right)^{T^{\prime}},\left(-a_{11} P^{*}+a_{21} Q^{*}\right)^{T^{\prime}}\right) .
$$

In view of the projective invariance of the intersection multiplicity [14] we have:

$$
\begin{aligned}
I_{p^{\prime}}\left(\left(a_{22} P^{*}-a_{12} Q_{22}^{*}\right)^{T^{\prime}}\right. & \left.,\left(-a_{11} P^{*}+a_{21} Q^{*}\right)^{T^{\prime}}\right) \\
& =I_{p}\left(\left(a_{22} P^{*}-a_{12} Q_{22}^{*}\right),\left(-a_{11} P^{*}+a_{21} Q^{*}\right)\right) .
\end{aligned}
$$

Since the equations $\phi=a_{22} P^{*}-a_{12} Q_{22}^{*}, \psi=-a_{11} P^{*}+a_{21} Q^{*}$ can be solved for $P^{*}, Q^{*}$ we have $I_{p^{\prime}}\left(P^{\prime *}, Q^{\prime *}\right)=I_{p}\left(P^{*}, Q^{*}\right)$ and the result follows. 


\subsection{A first application.}

In our first application we consider the family of quadratic Hamiltonian systems with a center. This family was studied in the literature [1], $[\mathbf{2}],[\mathbf{2 6}],[\mathbf{3 5}]$. Since the systems are Hamiltonian, there are no limit cycles and the bifurcation diagram is made up of bifurcation points of singularities and bifurcation of saddle to saddle connections. We give below the proof of a unified characterization (a part of this result was stated in [32] but given there without proof) of the bifurcation locus for this family. The changes in the phase portraits are governed by the changes in the singular invariant cubics. These curves are of two types: simple and multiple, according to the definitions given below:

Definition 4.2. Let us consider a structured system $(S, f)$ where $S$ is given by equations (3.3) and $C$ is an invariant algebraic curve $C: f=0$. We say that this curve is a simple singular invariant curve if it is irreducible, all its singularities are ordinary double points located in the finite plane and the curve is transversal to the line at infinity.

Definition 4.3. A singular invariant algebraic curve $C_{0}$ of a family $F$ of systems $S_{\lambda}$ of degree $n$ given by equations (3.3), depending continuously on the multiparameter value $\lambda \in \mathbb{R}^{s}$, which corresponds to the value $\lambda_{0} \in \mathbb{R}^{s}$ of the parameter, is a multiple invariant algebraic curve of multiplicity $m$ for the family, if and only if in for any $\varepsilon>0$ there is a neighborhood $V$ of Sing $\omega_{\lambda_{0}} \cap \widetilde{C}_{0}$ such that for $\lambda$ with $\left|\lambda_{0}-\lambda\right|<\varepsilon$ there are systems $S_{\lambda}$ which have exactly $m$ simple invariant algebraic curves $C_{\lambda, 1}, C_{\lambda, 2}, \ldots, C_{\lambda, m}$, with $V \supset\left(\operatorname{Sing} \omega_{\lambda} \cap \widetilde{C}_{\lambda, i}\right)$ and no such system $S_{\lambda}$ has more than $m$ such curves.

Notation 4.1. We denote the multiplicity of a multiple singular invariant cubic curve $C$ of a family $F$ of systems $S$ given by equation (3.3) by $m(C, F)$ and when the meaning is clear we use the shorthand $m(C)$.

The bifurcation diagram of the family of quadratic Hamiltonian systems with a center is determined by the multiple singular invariant cubic curves. To see this we place the center at the origin and use affine linear transformations on $x, y$ and positive time rescaling to bring the systems to the following canonical form:

$$
\begin{aligned}
& d x / d t=-y+k x^{2}+n y^{2}, \\
& d y / d t=x+a x^{2}-2 k x y .
\end{aligned}
$$

We have the following Hamiltonian:

$$
H_{\lambda}(x, y)=-\left(a x^{3}-n y^{3}\right) / 3+k x^{2} y-\left(x^{2}+y^{2}\right) / 2
$$


where $\lambda=(a, k, n)$. Since we are interested only in nonlinear systems, we must have $(a, k, n) \neq 0$ and hence the systems can be rescaled using homotheties of the axes. Thus the parameter space is actually the real projective plane $\mathbb{R} P(2)$. This condenses the bifurcation diagram on a disk with the identification of opposite points on the circumference. We may place $n=0$ as the line at infinity of $\mathbb{R}^{2}$, i.e. the circumference of the disk. We observe that the following identity holds for the systems (QHC):

$$
H_{(-a, k, n)}(x, y)=H_{(a, k, n)}(-x, y) .
$$

Thus discussing the systems (QHC) in a semidisk corresponding to a $\leq 0$ is sufficient.

The systems (QHC) are Hamiltonian, hence they do not have any limit cycles and the bifurcations of the family are of just of two types: bifurcations of singularities and bifurcations of saddle to saddle connections. Bifurcations in the number of singularities occur when one or more singularities coalesce in the finite plane or at infinity or when singularities in the finite plane disappear at infinity.

Notation 4.2. Let $N(\lambda)$ be the number of singular points in the finite plane of the system (QHC) for the parameter value $\lambda$.

The singularities of the system (QHC) are the common zeroes of the curves:

$$
\begin{aligned}
& P(x, y)=-y+k x^{2}+n y^{2}=0, \\
& Q(x, y)=x+a x^{2}-2 k x y=0 .
\end{aligned}
$$

We denote by $P^{*}$ and $Q^{*}$ the polynomials defining the projective completions of these curves i.e.:

$$
\begin{aligned}
& P^{*}(x, y, z)=-y z+k x^{2}+n y^{2}=0, \\
& Q^{*}(x, y, z)=x z+a x^{2}-2 k x y=0 .
\end{aligned}
$$

If the curves $P^{*}=0$ and $Q^{*}=0$ intersect at infinity, we have fewer singularities in the finite plane so in this case $N(\lambda) \leq 3$. This also happens when the curves intersect in the finite plane with multiplicity greater than one. Since the second curves in (4.7) and (4.8) are reducible, we easily obtain the conditions for this to happen. We have the two finite singularities $(0,0),(0,1 / n)$ if $n \neq 0$ and the two other singularities must satisfy $1+a x-2 k y=0$ and $-y+k x^{2}+n y^{2}=0$. If $a \neq 0$, replacing $x=(2 k y-1) / a$ in the second equation and we obtain the equation in $y$ :

$$
\left(n a^{2}+4 k^{3}\right) y^{2}-\left(a^{2}+4 k^{2}\right) y+k=0 .
$$


If $a=0 \neq k$, replacing $y=1 /(2 k)$ in the first equation of (4.5) we get the equation

$$
k x^{2}+(n-2 k) /\left(4 k^{2}\right)=0 .
$$

Notation 4.3.

$$
\begin{aligned}
\delta & =a^{2}-4 k n+8 k^{2}, \\
C & =n a^{2}+4 k^{3} .
\end{aligned}
$$

The discriminant of the equation (4.9) is $a^{2} \delta$. If $n C \delta(n-2 k) \neq 0$ and if $a \neq 0$ we can solve the equation (4.9) for $y$ while if $a=0 \neq k$ and we can solve the equation (4.10) for $x$ to obtaining the two remaining singularities. Equation (4.9) has two distinct real solutions if and only if $\delta>0$. Equation (4.10) applies in case $a=0$ in which case $\delta=-4 k(n-2 k)$. So (4.9) (or (4.10)) has two distinct real solutions if and only if $\delta>0$. Thus all nonlinear systems (QHC) have at least two singularities and we have:

$$
N(\lambda)= \begin{cases}4 \quad & \text { iff } n C \delta(n-2 k) \neq 0 \text { and } \delta>0 \\ 3 \quad & \text { iff one and only one of the equations } \\ & n=0, \delta=0, C=0, n-2 k=0 \text { is satisfied. } \\ 2 \quad & \text { iff two distinct equations } \\ & n=0, \delta=0, C=0, n-2 k=0 \text { are satisfied or } \delta<0\end{cases}
$$

The function $N$ is discontinuous at all points of the curve

$$
C_{1}: n C \delta(n-2 k)=0
$$

and hence this is a bifurcation curve of singularities.

The systems (QHC) also have bifurcation of saddle to saddle connections. It is clear from the equations (QHC) that if $a=0 \neq k, 1-2 k y=0$ is an invariant line and on this line lie two singularities of the systems which are real if $\delta>0$. Hence the set of points on $a=0<\delta$ is part of the bifurcation set of saddle to saddle connections. If $a \neq 0$, to have a saddle to saddle connection, two singular points of the systems must be on the same level curve $H(x, y)=K$ where $K$ is a constant. These must also be singular points of this curve, so this curve is reducible and hence the system must have an invariant straight line which clearly cannot pass through the origin which is a center. We may assume that this line is of the form: $f(x, y)=r x+s y+1 / n=0$, for $n \neq 0$. We must have: 


$$
\begin{gathered}
P \partial f / \partial x+Q \partial f / \partial y=(r x+s y+1 / n)\left(r^{\prime} x+s^{\prime} y+t^{\prime}\right) . \\
r\left(-y+k x^{2}+n y^{2}\right)+s\left(x+a x^{2}-2 k x y\right) \\
=(r x+s y+1 / n)\left(r^{\prime} x+s^{\prime} y+t^{\prime}\right)
\end{gathered}
$$

Identifying coefficients we get $t^{\prime}=0, r^{\prime}=s n, s^{\prime}=-r n$ and replacing in the remaining equations we get $s=-1, r=a /(k+n)$, (if $k+n \neq 0)$ yielding the invariant line: $(a /(n+k)) x-y+1 / n=0$ and the condition the parameters must satisfy:

$$
a^{2} n+(k+n)^{2}(2 k-n)=0
$$

We note that the line $f=0$ passes through the singularity $(0,1 / n)$.

Thus the bifurcation points of saddle to saddle connections are situated on the curve:

$$
C_{2}: a[a n-(n+k)(n-2 k)]=0
$$

and in case $a=0$ for $\delta>0$. Except for points on the curve

$$
C: n C \delta(n-2 k) a[a n-(n+k)(n-2 k)]=0
$$

there are no other bifurcation points $[\mathbf{2 6}]$. We shall denote by $\operatorname{Sing}(C)$ the set of the real singular points of the algebraic curve $C$.

Phase portraits do not change on each one of the components of $C \backslash \operatorname{Sing}(C)$ as one could see in the bifurcation diagram [26] given in Figure 1. We call these points, codimension one bifurcation points of the family. All points $\lambda$ on $\operatorname{Sing}(C)$ are bifurcation points on any smooth curve (in the parameter space) passing through $\lambda$. We call these codimension two bifurcation points of the family. $\lambda$ is a bifurcation point of singularities of the family (QHC) if and only if it belongs to $C_{1}$.

The bifurcation locus is thus described as the union of bifurcation points of singularities in the finite plane $((n-k) \delta=0)$, of bifurcation points of singularities at infinity $(n C=0)$ and of bifurcation of saddle to saddle connections $\left(C_{2} \backslash\{\delta<0\}\right)$. We give below a unified characterization of the bifurcation locus $C \backslash\{\delta<0\}$ in terms of the multiple singular invariant curves for the family of systems (cf. Definition 4.3): 
D. Schlomiuk

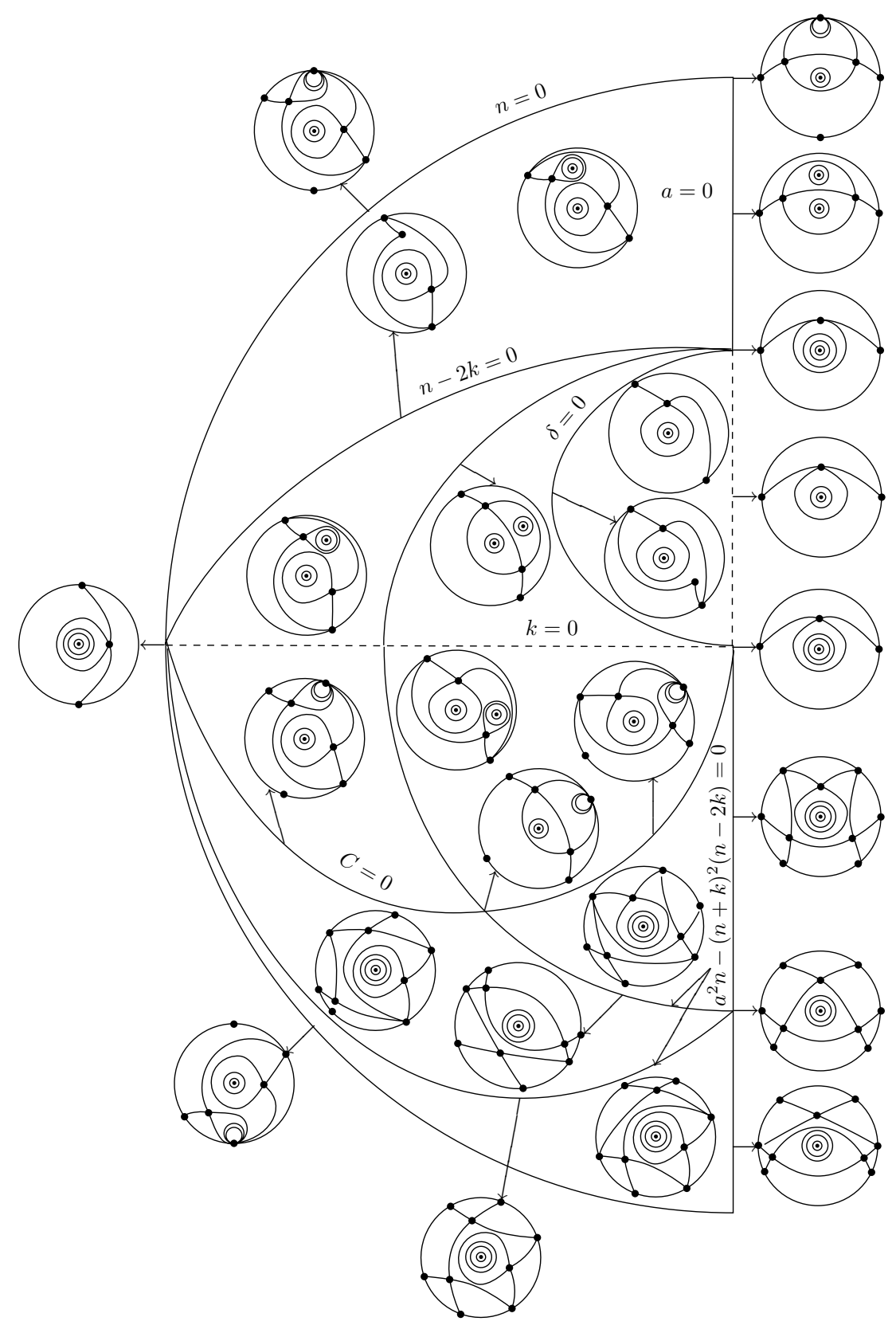

Figure 1 
Theorem 4.1. $\lambda$ is a bifurcation point of the family of quadratic Hamiltonian systems with a center if and only if the vector field $v_{\lambda}=P_{\lambda} \partial / \partial x+Q_{\lambda} \partial / \partial y$ in this family has a multiple invariant cubic curve $C_{\lambda}$ and in this case we have $2 \leq m\left(C_{\lambda}\right) \leq 3 . \lambda$ is a codimension one bifurcation point of the family $(Q H C)$ if and only if $m\left(C_{\lambda}\right)=2$ and there is no cubic curve with $m\left(C_{\lambda}\right)=3 . \lambda$ is a codimension two bifurcation point of the family if and only if there is a curve $C_{\lambda}$ with $m\left(C_{\lambda}\right)=3$. Furthermore, the multiplicity $m\left(C_{\lambda}\right)$ of the above curves within the family coincides with $\mu\left(C_{\lambda}\right)$ and we thus have:

$$
m\left(C_{\lambda}\right)=\mu\left(C_{\lambda}\right)=\sum_{p \in \underset{p \in \operatorname{Sing}\left(\omega_{\lambda}\right)}{p \in C_{\lambda}}} I_{p}\left(P_{\lambda}^{*}, Q_{\lambda}^{*}\right) .
$$

Proof: We may restrict ourselves to the family (QHC). If a bifurcation value $\lambda_{0}$ of the parameter belongs to $C_{2} \backslash\{\delta<0\}$, it is a saddle to saddle connection and then we have a reducible invariant cubic which is clearly not simple. For all bifurcation points of saddle to saddle connections which are not in $\operatorname{Sing}(C)$, we have four singular points in the finite plane, all hyperbolic and two of these are saddles located on the reducible cubic. In any neighborhood of $\lambda_{0}$ we have points for which we no longer have a saddle to saddle connection and since the singular points are stable by perturbation, each perturbed saddle points sits on a simple singular cubic in the perturbed system. So the statement holds for all such $\lambda_{0}$. If $\lambda_{0}$ is on $n C=0$, the system has an invariant curve which is tangent to the line at infinity. Indeed, on $n=0$, the curves $P^{*}=0$ and $Q^{*}=0$ intersect at $z=0=x$. The invariant cubic curves are given by the equation: $-a x^{3} / 3+k x^{2} y-\left(x^{2}+y^{2}\right) z / 2=K z^{3}$. All these curves are tangent to the line at infinity and among them we have two nodal cubics with real tangents. Let $C_{\lambda}$ be one of these. Since $I_{[0,1,0]}\left(P_{\lambda}^{*}, Q_{\lambda}^{*}\right)=1$ we have $\mu\left(C_{\lambda}\right)=2$. In a neighborhood of $\lambda_{0}$ there are values $\lambda$ for which $P_{\lambda}^{*}=0, Q_{\lambda}^{*}=0$ no longer intersect at infinity. The point $[0,1,0]$ produces in a general quadratic perturbation an additional singular point in the finite plane (a center, yielding a simple singular invariant cubic). So $m\left(C_{\lambda}\right)=2$. If $C=0$, replacing this in the equation (4.9) we obtain

$$
-\left(a^{2}+4 k^{2}\right) y+k=0
$$

and hence $y=k /\left(a^{2}+4 k^{2}\right)$ and $x=(2 k y-1) / a$ yielding a singular point $(x, y)=\left(-\left(a^{2}+2 k^{2}\right) /\left(a^{2}+4 k^{2}\right), k /\left(a^{2}+4 k^{2}\right)\right)$. The cubic passing through this point is singular and it is tangent to the line at infinity and we have an analogous situation to the case $n=0$.

If for $\lambda_{0}$ we have $\delta(n-2 k)=0$ and $\lambda_{0} \notin \operatorname{Sing}(C)$ then the systems have a cusp. Indeed, if $n-2 k=0$, this cusp is located at $(0,1 / n)$, the curve 
$H(x, y)=H(0,1 / n)$ being a cubic with identical tangents at this point hence a multiple cubic for the family. In a quadratic perturbation this singular point splits into two singularities yielding two simple invariant cubics in the perturbation so $m\left(C_{\lambda_{0}}\right)=2$. At the same time we have $\mu\left(C_{\lambda_{0}}\right)=2$, the only singular point of the system which is located on both $P_{\lambda_{0}}^{*}=0, Q_{\lambda_{0}}^{*}=0$ being $(0,1 / n)$ and $I_{(0,1 / n)}\left(P_{\lambda_{0}}^{*}, Q_{\lambda_{0}}^{*}\right)=2$. Hence $\mu\left(C_{\lambda_{0}}\right)=2$. An analogous situation occurs on $\delta=0$ where we have an invariant cubic with a finite cusp: $\left(a(k-n) / C,\left(4 k^{2}+a^{2}\right) / C\right)$. This cuspidal cubic splits in a general quadratic perturbation in two nodal cubics. To obtain the second part of the proof we first observe that

$$
\operatorname{Sing}(C)=\{[0: 1: 0],[0: 1: 2],[1: 0: 0],[0: 0: 1],[0: 1:-1],[ \pm \sqrt{2}: 1:-2]\}
$$

If the parameter $\lambda$ is one of the three points $[0: 0: 1],[0: 1: 2],[1: 0: 0]$ we have the same phase portraits. In all three cases we have two finite singularities, in the first two cases they are the origin and $(0,1 / n)$ and in the third case the origin and $(-1 / a, 0)$. The curves $H(x, y)=$ $H(0,1 / n)$ in the first two cases are of multiplicity three for the family because these curves pass through the point at infinity $y=0=z$ and because $I_{[1,0,0]}\left(P^{*}, Q^{*}\right)=2$. Hence in a perturbation the curve $H(x, y)=H(0,1 / n)$ splits into three curves, two passing through the two points which bifurcate in the finite plane from the point at infinity $y=0=z$ and one passing through the point $(0,1 / n)$ which exists for the entire family when $n \neq 0$. We clearly have $\mu\left(C_{\lambda_{0}}\right)=3$ since $I_{[1,0,0]}\left(P^{*}, Q^{*}\right)=2$ and $I_{[0,1 / n]}\left(P^{*}, Q^{*}\right)=1$. Thus $m\left(C_{\lambda}\right)=\mu\left(C_{\lambda}\right)=3$ in this case. Analogous arguments hold for the point $[1,0,0]$. The cases $\lambda=[0: 1: 0]$ and $\lambda=[ \pm \sqrt{2}: 1:-2]$ are analogous and we shall only consider the first case. In this case the system has two hyperbolic saddles: $(1 / \pm \sqrt{2}, 1 / 2)$ lying on the invariant line $1-2 y=0$ and an invariant parabola whose point at infinity is $x=0=z$. For this point we have $I_{[0,1,0]}\left(P^{*}, Q^{*}\right)=1$ and hence in a perturbation of the systems this point produces a finite hyperbolic singularity and we have in such a perturbation three simple singular cubic curves $H(x, y)=K$ with singularities in the neighborhood of the reducible cubic whose components are the invariant line and the parabola. We again have $m\left(C_{\lambda}\right)=\mu\left(C_{\lambda}\right)=3$.

Remark 4.1. The result above characterizes the bifurcation locus in terms of multiplicity of singular invariant cubics only. This is a unified way of treating the bifurcation points of singularities, be they finite or infinite and bifurcations of saddle-to-saddle connection by using global properties of the systems, in this case the existence of a multiple invariant singular cubic curves for the family. 
Concluding comments and problems. In the above application the bifurcation points are classified by the number $m(C)(=\mu(C))$ of simple singular invariant curves which can bifurcate from an invariant multiple curve $C$ in a quadratic perturbation. Although this result is a very limited one, being done for a very particular class of systems, it does however tell us in a sort of a bird's eye view, what happens globally to the systems on the bifurcation diagram, without any need of coordinates. This raises the problem of attempting to go from just drawing bifurcation diagrams and directories of inequalities with corresponding phase portraits to an effort to sum up informations in bifurcation diagrams for nonintegrable systems. The analysis of singularities for such classes is much more complex but the same kind of analysis must be performed, to have a clear algebro-geometric synthesis, for example for the class of quadratic systems with a focus, the class involved in Hilbert's 16th problem. Of course, for the bifurcation diagram to be complete we need to handle the more difficult problem of limit cycles. While this problem will essentially involve tools of analysis, we suspect that the role of algebro-geometric concepts is not yet over.

Finally we consider the following two problems:

In the above application, at bifurcation points some global "accidents" occur. In analogous fashion we may try to define "global accidents" (specific local and nonlodal accidents would enter into the description of such global accidents) for quadratic systems and to assign a global measure to them (like $m\left(C_{\lambda}, F\right)=\mu\left(C_{\lambda}, S_{\lambda}\right)$ in the above application). For this to be done, the geometry of the parameter space of the quadratic systems as well as of the bifurcation hypersurfaces must play a role as it does in this work. With few exceptions (such as this one or [26]) this has not been done as the reader may easily see by consulting the literature (for example [1], [35], etc.).

Another question is the problem of generalizing the above result for families of systems with a polynomial first integral of a given degree or to families of systems which are algebraically integrable with generic invariant algebraic curves of a certain degree.

\subsection{A second application.}

Our second application concerns more geometrical formulations of theorems by using concepts introduced in the preceding section. We give but one example: a theorem about Darboux integrability of planar systems proved by Christopher and Kooij [20] which the author state as follows:

Theorem 4.2 (Kooij and Christopher [20]). Consider a poly- 
nomial system given by equation (3.3) where $\max (\operatorname{deg}(P), \operatorname{deg}(Q))=n$. Assume this system has $k$ algebraic invariant algebraic curves $C_{i}: f_{i}(x, y)=0, i=1, \ldots, k,\left(f_{i}(x, y)\right.$ polynomials with complex coefficients). We assume that the curves $C_{i}$ satisfy the following conditions:

i) All curves $C_{i}$ are irreducible and such that their highest terms have no repeated factors,

ii) no more than two curves meet at any point in the finite plane and are not tangent at these points,

iii) no two curves have a common factor in their highest order terms,

iv) the sum of the degrees of the curves is $n+1$.

Then the system has an integrating factor of the form $1 /\left(f_{1} f_{2} \ldots f_{k}\right)$ and a Darboux first integral of the form $f_{1}^{\lambda_{1}} f_{2}^{\lambda_{2}} \ldots f_{k}^{\lambda_{k}}$ where $\lambda_{i}$ are complex constants.

Remark 4.2. We note that two of the hypotheses are stated using the expression "no repeated factor" and the statement of the theorem separates the finite intersections from the infinite ones.

To restate the theorem in more geometric terms, we shall use the following definition:

Definition 4.4. Two projective curves $F=0$ and $G=0$ in $\mathbb{C} P(2)$ intersect transversely at a point $p$ in $\mathbb{C} P(2)$ if and only if $I_{p}(F, G)=1$.

Theorem 4.3 (restated using intersection multiplicities). Consider a polynomial system given by equation (3.3) where $\max (\operatorname{deg}(P), \operatorname{deg}(Q))=n$. Assume this system has $k$ algebraic invariant algebraic curves $C_{i}: f_{i}(x, y)=0, i=1, \ldots, k$. We assume that the curves $C_{i}$ satisfy the following conditions:

i) All curves $C_{i}$ are irreducible.

ii) Let $C_{1}^{*}, C_{2}^{*}, \ldots, C_{k}^{*}$ be the projection completions in $\mathbb{C P}(2)$ of the curves $C_{i}$. Then two distinct curves of the curves $C_{1}^{*}, C_{2}^{*}, \ldots, C_{k}^{*}$, $Z=0$, intersect transversely and three distinct such curves do not have a common point.

iii) The sum of the degrees of the curves is $n+1$.

Then the system has an integrating factor of the form $1 /\left(f_{1} f_{2} \ldots f_{k}\right)$ and a Darboux first integral of the form $f_{1}^{\lambda_{1}} f_{2}^{\lambda_{2}} \ldots f_{k}^{\lambda_{k}}$ where $\lambda_{i}$ are complex constants.

Clearly the Theorem 4.3 as stated above, gained more clarity, unity and transparence of geometrical meaning. 


\section{References}

1. E. A. Andronova, Decomposition of the parameter space of a quadratic equation with a singular point of center type and topological structures with limit cycles, Ph. D. Thesis (in russian), Gorky, Russia (1988).

2. J. C. ARtÉs and J. Llibre, Quadratics Hamiltonian vector fields, J. Differential Equations 107 (1994), 80-95.

3. A. Borel, On the development of Lie group theory, L. E. J. Brower Memorial Lecture, Nieuw Arch. Wisk. (3) 27 (1979), 13-25.

4. C. Самасно, Complex foliations arising from polynomial differential equations, Notes by Maria Izabel Camacho, in "Bifurcations and Periodic Orbits of Vector Fields," (D. Schlomiuk, ed.), Kluwer Academic Publishers, 1993.

5. C. Camacho, A. Lins Neto and P. Sad, First integrals of algebraic differential equations, Lecture Notes for participants to the School on Dynamical Systems, ICTP, Trieste (1992).

6. M. M. CARnicer, The Poincaré problem in the nondicritical case, Ann. of Math. 140 (1994), 289-294.

7. D. Cerveau and A. Lins Neto, Holomorphic foliations in $C P(2)$ having an invariant algebraic curve, Ann. Inst. Fourier (Grenoble) 41 (1991), 883-903.

8. W. A. Coppel, A survey of quadratic systems, J. Differential Equations 2 (1966), 293-304.

9. G. Darboux, Mémoire sur les équations différentielles algébriques du premier ordre et du premier degré, Bull. Sci. Math. Sér. (2) 2(1) (1878), 60-96;123-144;151-200.

10. H. Dulac, Sur les cycles limites, Bull. Soc. Math. France 51 (1923), $45-188$.

11. F. Dumortier, R. Roussarie and C. Rousseau, Hilbert's 16th problem for quadratic systems, J. Differential Equations 110(1) (1994), 86-133.

12. F. Dumortier, R. Roussarie and C. Rousseau, Elementary graphics of cyclicity 1 and 2, Nonlinearity 7 (1994), 1001-1003.

13. J. ECALLE, Introduction aux fonctions analysables et preuve de la conjecture de Dulac, Hermann, Actualités Math. (1992).

14. W. Fulton, "Algebraic curves," W. A. Benjamin, Inc., 1969.

15. X. Gomez-Mont, On foliations in $\mathbb{C} P(2)$ tangent to an algebraic curve, Preprint. 
16. E. A. Gonzales Velaso, Generic properties of polynomial vector fields at infinity, Trans. Amer. Math. Soc. 143 (1969), 201-222.

17. Yu. S. IL'yashenko, "Finiteness theorems for limit cycles," Trans. Math. Monographs, A.M.S. 94, 1991.

18. J. P. JouAnolou, "Équations de Pfaff algébriques," Lecture Notes in Math. 708, Springer-Verlag, 1979.

19. F. KiRWAN, "Complex algebraic curves," London Mathematical Society Student Texts 23, Cambridge University Press, 1992.

20. R. E. Koolj And C. J. Christopher, Algebraic invariant curves and the integrability of polynomial systems, Appl. Math. Lett. 6(4) (1993), 51-53.

21. A. Lins Neto, Algebraic solutions of polynomial differential equations in dimension two, in "Holomorphic dynamics," Lecture Notes in Math. 708, Springer-Verlag, 1988, pp. 883-903.

22. B. MAzur, Fermat's Last Theorem, AMS-CMS-MAA Joint Invited Address, videotaped lecture (1993).

23. P. Painlevé, Mémoire sur les équations différentielles du premier ordre, Ann. Sci. École Norm. Sup. (3) 8 (1881), 9-58;103-140; 201-226;276-284;, (1882), 9-30;101-144;283-308.

24. P. Painlevé, Sur les intégrales algébriques des équations différentielles du premier ordre, C. R. Acad. Sci. Paris 110 (1890), 945-948.

25. J. PAL, Quadratic systems with a weak focus, Ph. D. Thesis, Université de Montréal (in preparation).

26. J. PAl AND D. Schlomiuk, Summing up the dynamics of quadratic hamiltonian systems with a center, Canad. J. Math. (to appear).

27. H. Poincaré, Sur l'intégration algébrique des équations differentielles, C. R. Acad. Sci. Paris 112 (1891), 761-764.

28. H. Poincaré, Sur l'intégration algébrique des équations differentielles du premier ordre et du premier degré, Rend. Circ. Mat. Palermo 5 (1891), 160-191.

29. D. Schlomiuk, Elementary first integrals and algebraic invariant curves of differential equations, Exposition. Math. 11 (1993), 433454.

30. D. Schlomiuk, Algebraic and geometric aspects of the theory of polynomial vector fields, in "Bifurcations and Periodic Orbits of Vector Fields," (D. Schlomiuk, ed.), Kluwer Academic Publishers, 1993, pp. 429-467.

31. D. Schlomiuk, Algebraic particular integrals, integrability and the problem of the center, Trans. Amer. Math. Soc. 338(2) (1993), 
799-841.

32. D. Schlomiuk, On the global analysis of the planar quadratic vector fields, in "Proceedings of the 2nd World Congress of Nonlinear Analysis," 1993.

33. C. Seidenberg, Reduction of singularities if the differential equation $A d y=B d x$, Amer. J. Math. 90 (1968), 248-269.

34. A. VAN DEN EsSEn, Reduction of singularities of the differential equation $A d y=B d x$, Lecture Notes in Math. 712, Springer-Verlag, 1975, pp. 44-59.

35. N. I. Vulpe, Affine-invariant conditions for the topological discrimination of quadratic systems with a center, (transl.), Differentsial'nye Uravneniya 19(3) (1983), 371-379.

\author{
Département de Mathématiques et de Statistique \\ Université de Montréal \\ C.P. 6128, Succursale Centre-Ville \\ Montréal (Québec), H3C 3J7 \\ CANADA \\ e-mail: dasch@mathcn.umontreal.ca
}

Primera versió rebuda el 30 de Novembre de 1996, darrera versió rebuda el 21 de Març de 1997 